\title{
Polarimetry of birefringent biological tissues with arbitrary fibril orientation and variable incidence angle
}

\author{
Félix Fanjul-Vélez* and José Luis Arce-Diego \\ Applied Optical Techniques Group, Electronics Technology, Systems and Automation Engineering Department, \\ University of Cantabria, Avenida de los Castros S/N, 39005 Santander, Cantabria, Spain \\ *Corresponding author: fanjulf@unican.es
}

Received December 3, 2009; revised February 11, 2010; accepted February 26, 2010; posted March 8, 2010 (Doc. ID 120923); published April 12, 2010

\begin{abstract}
Polarimetric optical techniques such as polarization microscopy or polarization-sensitive optical coherence tomography normally assume that light is perpendicular to the sample surface and that fibrils of a birefringent biological tissue are arranged in a plane parallel to this surface. The approaches that describe quantitatively polarimetric data from tissues with nonparallel fibril orientation and/or off-axis incidence usually lack a rigorous theoretical analysis. We present a polarimetric model with arbitrary fibril orientation and/or variable incidence angle by means of the extended Jones matrix theory, the polar decomposition, and Poincaré equivalence theorem. The model, suitable for diagnosis or tissue structure analysis, is applied to articular cartilage. (C) 2010 Optical Society of America

OCIS codes: 260.5430, 260.1440, 170.3880, 170.6935
\end{abstract}

Birefringent collagen-based biological tissues, such as tendons, ligaments, or cartilage [1], are particularly sensitive to polarization optical techniques, such as polarized light microscopy (PLM) or polarization-sensitive optical coherence tomography (PS-OCT). The quantitative evaluation of the results normally assumes that the incident radiation is perpendicular to the tissue surface and also that the collagen fibrils of the birefringent tissue lie in a plane parallel to this surface. Under such conditions the tissue is usually modeled by a Jones linear retarder. A further decomposition or numerical procedure extracts optic axis orientation and retardation. However, this approach is not valid when the incident radiation is not perpendicular to the tissue and/or when the fibril orientation of the collagen inside is arbitrary, such as in full-field PS-OCT on curved structures. Some authors have recently tried to model the polarimetric response in this case with a single layer crystal with only one propagation vector in the supposed uniaxial medium, and whose optic axis is constrained to a particular plane [2]. Others have used a model of propagation based on on-axis linear retarder Jones matrices and fibrils projections for nonnormal incidence [3]. Neither of these approaches represents a rigorous quantitative polarimetric analysis. An approach for off-axis propagation was proposed by $\mathrm{Gu}$ and Yeh [4] by means of the $2 \times 2$ extended Jones matrices. This method was used for the calculus of the propagation of light through two and three anisotropic layers in which the optic axis is constrained to be parallel to the layers, and in which the effects at the boundaries are not taken into account [5]. To the authors' knowledge previous works do not provide a rigorous model for the polarimetric response of birefringent collagen-based biological tissues. We propose in this Letter a polarimetric model of birefrigent biological tissues with arbitrary fibril orientation and variable incidence angle. This model employs the ex- tended Jones matrix theory, which considers only completely polarized light, the polar decomposition, and the Poincaré equivalence theorem.

The model we propose is based on the electromagnetic propagation of light in birefringent media. A birefringent collagen-based biological tissue can be modeled as a positive uniaxial crystal whose optic axis is coincident with the fibrils direction. A representation of this system is shown in Fig. 1. The birefringence is described by the extraordinary, $n_{e}$, and ordinary, $n_{o}$, refractive indexes and the orientation of the optic axis c. Propagation is modeled by vector $\mathbf{k}$ for the incident light in an isotropic medium and vectors $\mathbf{k}_{o}$ and $\mathbf{k}_{e}$ for the birefringent medium. The inci-

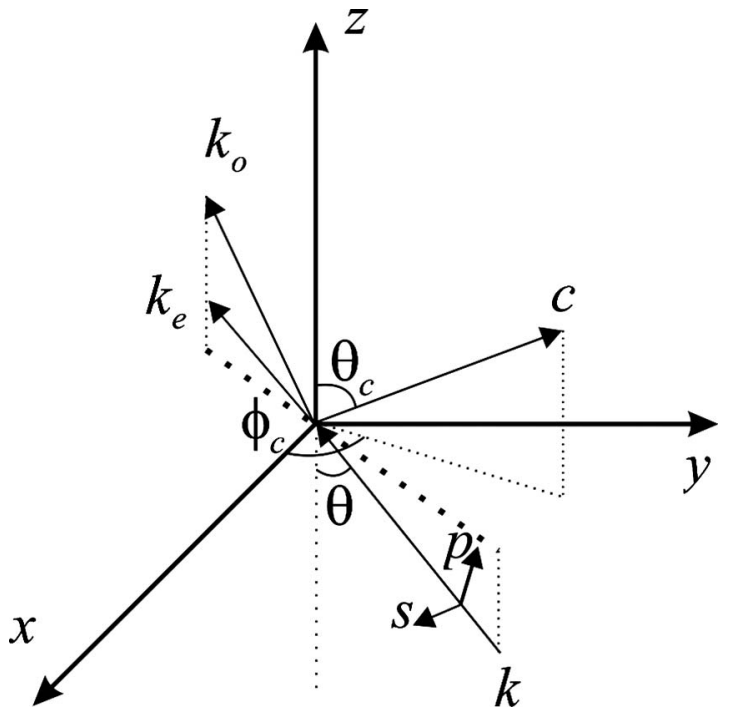

Fig. 1. Principal elements of the analysis. The layered birefringent material boundary is on the $X Y$ plane. $\mathbf{k}$ is the incident propagation vector; $\mathbf{k}_{o}$ and $\mathbf{k}_{e}$ are the propagation vectors inside the birefringent structure, $\mathbf{c}$ is the optic axis (polar angle $\theta_{C}$, azimuthal angle $\phi_{C}$ ), and $\mathbf{s}$ and $\mathbf{p}$ define the local polarization reference system. 
dent and output Jones vectors are referred to a system defined by TE and TM incident vectors $\mathbf{s}$ and $\mathbf{p}$, respectively. Modeling biological tissues as a parallel layered stack is convenient owing to the spatial variability of optical properties. Small birefringence is assumed, $\left|n_{e}-n_{o}\right| \ll n_{o}, n_{e}$. This is true for a great vari- ety of tissues, according to several experimental reports, with values of the order of $10^{-3}$ [6]. Dichroism, optical activity, and multiple reflections in the inner interfaces are neglected. The collagen-based birefringent $N$-layered biological tissue is described by the product of $2 \times 2$ extended Jones matrices [4]:

$$
\begin{aligned}
J_{\text {out }}= & M_{T} J_{\text {in }}=D_{\text {fin }} P_{N} T_{N-1, N} P_{N-1} T_{N-2, N-1} \ldots T_{2,3} P_{2} T_{1,2} P_{1} D_{i n i} J_{i n}=\left(\begin{array}{cc}
\mathbf{o} \cdot \mathbf{s} t_{s}^{\prime} & \mathbf{s} \cdot \mathbf{e} t_{s}^{\prime} \\
\mathbf{p}_{o} \cdot \mathbf{o} t_{p}^{\prime} & \mathbf{p}_{o} \cdot \mathbf{e} t_{p}^{\prime}
\end{array}\right)\left(\begin{array}{cc}
e^{-j k_{o z} d_{N}} & 0 \\
0 & e^{-j k_{e z} d_{N}}
\end{array}\right) \\
& \times\left(\begin{array}{ll}
\mathbf{o}_{N-1} \cdot \mathbf{o}_{N} & \mathbf{e}_{N-1} \cdot \mathbf{o}_{N} \\
\mathbf{o}_{N-1} \cdot \mathbf{e}_{N} & \mathbf{e}_{N-1} \cdot \mathbf{e}_{N}
\end{array}\right) \ldots\left(\begin{array}{ll}
\mathbf{s} \cdot \mathbf{o} t_{s} & \mathbf{p}_{o} \cdot \mathbf{o} t_{p} \\
\mathbf{s} \cdot \mathbf{e} t_{s} & \mathbf{p}_{o} \cdot \mathbf{e} t_{p}
\end{array}\right) J_{i n} .
\end{aligned}
$$

$J_{\text {in }}$ is the incident Jones vector; $J_{\text {out }}$ is the output Jones vector; $M_{T}$ is the extended Jones matrix of the biological tissue; matrices $D_{i n i}$ and $D_{\text {fin }}$ model the interaction at the interface between an isotropic medium (usually air) and the biological tissue; $P_{i}$ are propagation matrices through the $i$ th layer of depth $d_{i} ; T_{i, i+1}$ are the dynamical propagation matrices between uniaxial layers $i$ and $i+1$; $\mathbf{o}$ and $\mathbf{e}$ are the ordinary and extraordinary polarization vectors; $\mathbf{p}_{o}$ $=\mathbf{k}_{o} \mathbf{x s} ;$ and $t_{s}, t_{p}, t_{s}^{\prime}, t_{p}^{\prime}$ are the Fresnel transmission coefficients. The extended Jones matrix can be expressed as a product of a unitary matrix $U_{T}$ and a nonnegative definite hermitian matrix $H_{T}$, which contains the diattenuation effect, according to the polar decomposition,

$$
M_{T}=H_{T} U_{T} .
$$

The unitary matrix $U_{T}$ is further decomposed in the equivalent retarder and rotator according to the Poincaré equivalence theorem and the group theory. This allows the extraction of the optic axis orientation $\theta_{T}$ and retardation $\delta$ :

$$
U_{T}=R\left(-\theta_{T}\right) D(\delta) R\left(\theta_{T}\right) R\left(\theta_{R}\right) .
$$

Matrices $R(\theta)$ and $D(\delta)$ correspond to a general rotator of angle $\theta$ and a retarder of retardation $\delta$. The algorithm imposes that the optic axis is constrained to the interval $[0, \pi)$ (other orientations are equivalent) and retardation to be $[0, \pi / 2]$ to avoid ambiguities.

The previous model is applied to the polarimetric response of hyaline articular cartilage, a collagenbased biological tissue present in joints between bones, such as in the knee. Its degradation provokes disabilities like osteoarthritis. The structure of articular cartilage changes with depth from the articulating surface to the subchondral bone [7]. The configuration is represented in Fig. 2. The superficial or tangential zone contains collagen fibrils that are parallel to the tissue surface. In the middle or transitional zone fibrils bend like an arcade toward a perpendicular position. The base of this arcade is in the deep zone, where fibrils are totally perpendicular to the tissue surface. The arcade geometry in the middle layer is modeled by a superposition of layers in which the fibril direction is the tangent to the arcade. The thicknesses of the superficial, middle, and deep layers are $100 \mu \mathrm{m}, 500 \mu \mathrm{m}$ and $400 \mu \mathrm{m}$, respectively. The assumed birefringence is $5 \times 10^{-3}$, according to some experimental measurements $[8,9]$. Polar angle $\theta$ of the incident radiation vector $\mathbf{k}$ is varied in the analysis. The final results are referred to $X^{\prime} Y^{\prime} Z^{\prime}$ reference system. The cumulative retardation and slow-axis orientation as a function of depth for several incident radiation polar angles are presented in Figs. 3 and 4, respectively. These results could be obtained by a backscattering tomographic technique such as PS-OCT, in which optical activity would be low or no significant owing to compensation. In Fig. 3 (a) the cumulative retardation for perpendicular incident radiation is represented in solid black. In the first layer $(100 \mu \mathrm{m})$, the retardation increases with depth. The apparently decreasing retardation over $90^{\circ}$ is a consequence of the algorithm employed, as explained above. The graph presents constant periodicity in this layer, so it is composed by equally ori-

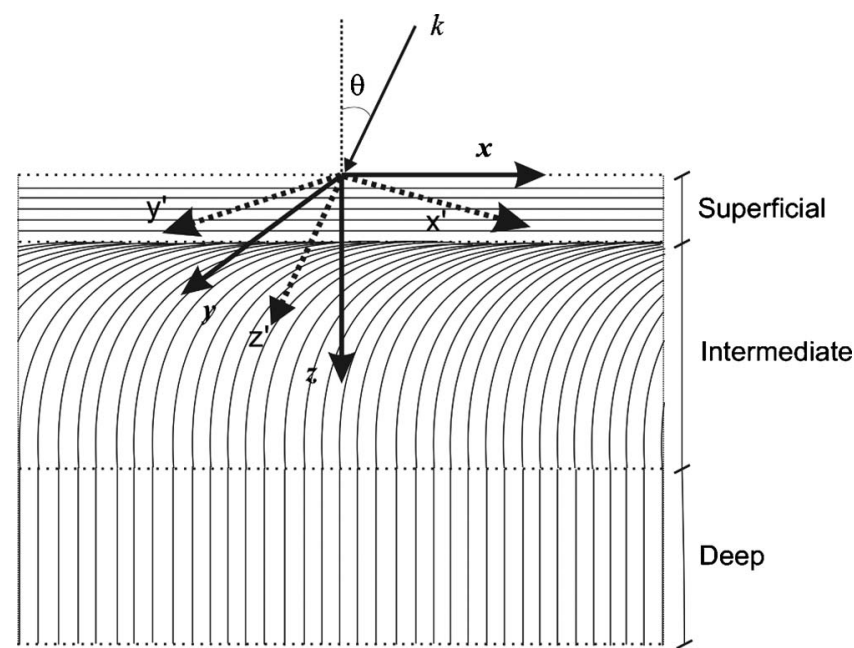

Fig. 2. Preferred collagen orientation of the articular cartilage. $\theta$ is the polar angle of the incident $\mathbf{k}$ vector. Representation reference system is $X^{\prime} Y^{\prime} Z^{\prime}$, where $Z^{\prime}$ is coincident with the $\mathbf{k}$ vector and $X^{\prime}$ is perpendicular to $\mathbf{y}$ vector. 

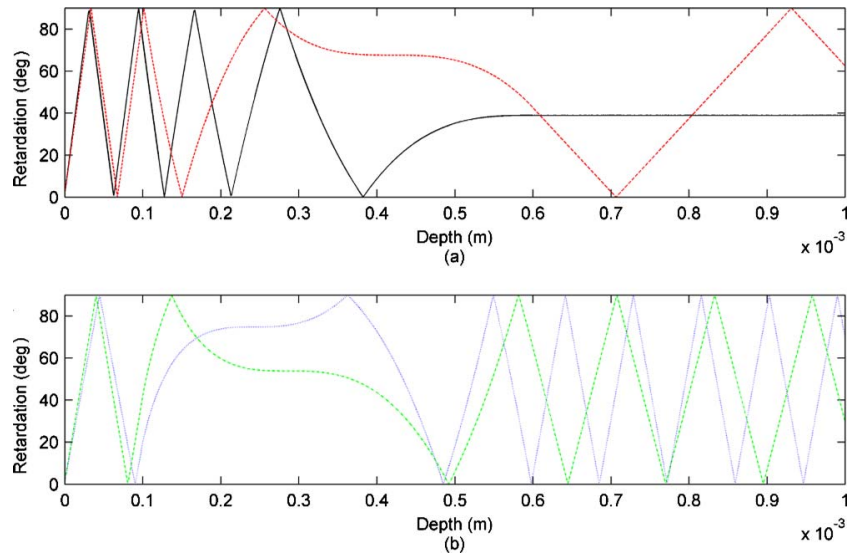

Fig. 3. (Color online) Retardation (deg) as a function of depth for several incident radiation polar angles: (a) polar angles $0^{\circ}$ (solid black) and $30^{\circ}$ (dashed red), (b) $60^{\circ}$ (dashed-dotted green) and $80^{\circ}$ (dotted blue).

ented fibrils. The period of the retardation oscillation increases in the intermediate layer. This fact shows that the fibrils are changing to a position of lower birefringence. Retardation increase is reduced until it remains constant at the beginning of the deep layer at $600 \mu \mathrm{m}$. This means that the last layer has no birefringence, so the radiation is incident following the optic axis. These results are in agreement with the structure previously described. The other graphs in Figs. 3(a) and 3(b) contain the results for polar angles $30^{\circ}, 60^{\circ}$, and $80^{\circ}$. In the first layer the retardation increase is lower as the polar angle increases. This implies that the radiation approaches the optic axis of the crystal. The constant period indicates that the fibrils orientation is also constant with depth. In the deep layer the birefringence increases with increasing polar angles, opposite to the previous case. Both results are in agreement with the expected behavior. Finally, in the intermediate layer the retardation decreases at the beginning of the layer, and it continues to decrease for the three cases $\left(30^{\circ}, 60^{\circ}\right.$, and $\left.80^{\circ}\right)$. This means that the fibrils orientation is changing
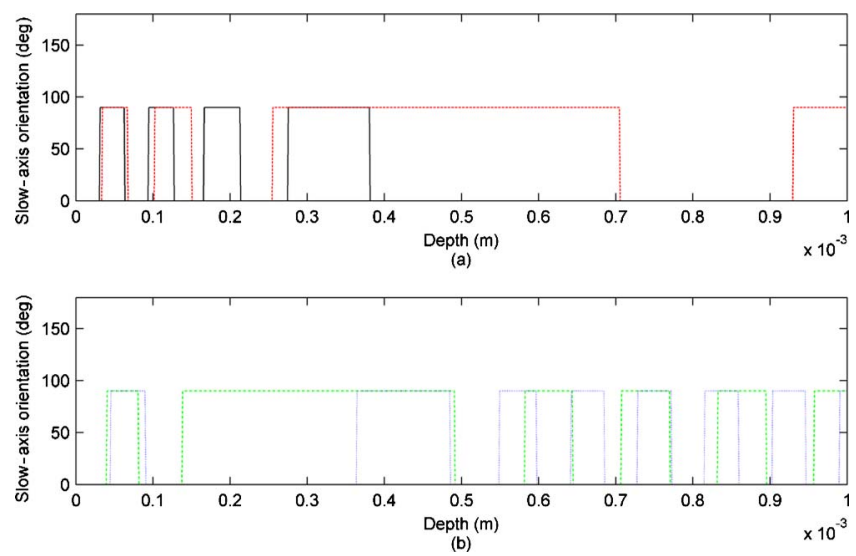

Fig. 4. (Color online) Slow-axis orientation (deg) as a function of depth for varying incident radiation polar angles: (a) polar angles $0^{\circ}$ (solid black) and $30^{\circ}$ (dashed red), (b) $60^{\circ}$ (dashed-dotted green) and $80^{\circ}$ (dotted blue). with depth and tends to be parallel to the incident radiation. When this occurs the birefringence is zero, and so the retardation remains constant (horizontal retardation tangent). This point tends to be situated at smaller depths when the incident polar angle is bigger. This is the expected result, since the fibril orientation at this layer will reach a position parallel to the radiation quicker when the polar angle is bigger. After that point, the retardation increases again. That means that the fibrils are changing to nonparallel to the incident radiation orientation. The slowaxis results can be seen in Fig. 4 for the same polar angles: (a) $\left(0^{\circ}\right.$ and $\left.30^{\circ}\right)$ and (b) $\left(60^{\circ}\right.$ and $\left.80^{\circ}\right)$. The slow axis is zero for all the polar angles and changes abruptly to $90^{\circ}$ at depth positions where the retardation is over $90^{\circ}$. The $90^{\circ}$ change is due to the Poincaré equivalence theorem and group theory algorithm, so the real optic axis orientation is zero for all depths and polar angles. With the proposed geometry (see Fig. 2) the projection of the optic axis on a plane perpendicular to the incident radiation would be a line in the $X Z$ plane. The $X^{\prime}$ axis of the coordinate system of the incident radiation is in this plane, and so the apparent fibril orientation azimuth angle is zero.

In summary, we introduce for the first time to our knowledge the analysis of the polarimetric response of birefringent biological tissues by means of extended Jones matrices, the polar decomposition, and the Poincaré equivalence theorem as described in group theory. Previous approaches do not treat appropriately tissues with nonnormal incidence and/or nonparallel to the surface fibrils. This analysis permits the calculation of a predictive polarization response of a biological tissue. This can be useful for data interpretation of polarization techniques, such as PLM, PS-OCT, or full-field PS-OCT. It can be employed for tissue diagnosis or tissue structure analysis, mainly collagen fiber orientation, and also in tissue engineering.

This work was partially supported by the project TEC2006-06548/TCM of the Spanish Ministry of Education and Science.

\section{References}

1. P. Fratzl, Collagen: Structure and Mechanics (Springer, 2008).

2. N. Ugryumova, J. Jacobs, M. Bonesi, and S. J. Matcher, Osteoarthritis Cartilage 17, 33 (2008).

3. M. C. van Turnhout, S. Kranenbarg, and J. L. van Leeuwen, J. Biomed. Opt. 14, 054018 (2009).

4. C. Gu and P. Yeh, J. Opt. Soc. Am. A 10, 966 (1993).

5. R. A. Farrell, D. Rouseff, and R. L. MacCally, J. Opt. Soc. Am. A 22, 1981 (2005).

6. Q. Zhou and R. W. Knighton, Appl. Opt. 36, 2273 (1997).

7. K. A. Athanasiou, E. M. Darling, and J. C. Hu, Articular Cartilage Tissue Engineering (Morgan \& Claypool, 2010).

8. T. Xie, S. Guo, J. Zhang, Z. Chen, and G. M. Peavy, J. Biomed. Opt. 11, 064001 (2006).

9. N. Ugryumova, S. V. Gangnus, and S. J. Matcher, Opt. Lett. 31, 2305 (2006). 